\title{
ON THE MANY-BODY VAN DER WAALS BINDING ENERGY OF A DENSE FLUID
}

\author{
B. R.A. NIJBOER \\ Institunt loor Theoretische Fysica, Rijksuniversiteit Utrecht, \\ Utrech, The Netherlands
}

Received 13 November 1974

\section{Synopsis}

We consider a dense system of neutral atoms. When the atoms are represented by isotropic oscillators (Drude-Lorentz model) interacting with nonretarded dipole-dipole forces, the binding energy of the system is given cxactly by a well-known expression which is written as a sum of two-bond, three-bond, etc., Van der Waals interactions. For a Bravais lattice this expression for the binding energy can be computed numerically to arbitrary accuracy. This has been done for the f.c.c. lattices of the noble-gas solids by Lucas. For a fluid an exact evaluation would require the knowledge of higher-order molecular distribution functions. Various approximations are discussed for this case, the simplest of which is the so-called long-wavelength approximation due to Doniach. When this approximation is checked by comparison with the exact result for it lattice, it turns out that the two-bond contribution leads to a value which is more than twice too large. Some more refined approximations are considered which treat the two-bond contribution exactly. It is pointed out that the model is consistent only if the distance of closest approach between the atoms is not too small.

1. Introduction. Let us consider a macroscopic dense system of neutral atoms. One might think for instance of a liquid or solid consisting of noble-gas atoms. In order to derive an expression for the many-body Van der Waals interaction cnergy of such a system we represent an atom by an isotropic harmonic oscillator (Drude-Lorentz model), where the electron (charge -e) oscillates with eigenfrequency "' with respect to the nucleus which is supposed to be kept in fixed position. The atoms are assumed to interact with electrostatic dipole-dipole interactions.

The hamiltonian for this system is

$$
H=\sum_{a=1}^{N}\left(\boldsymbol{p}_{a}^{2} / 2 m\right)+\sum_{a=1}^{N} \frac{1}{2} m \omega \omega_{0}^{2} \boldsymbol{u}_{a}^{2}+\frac{1}{2} e^{2} \sum_{a=1}^{N} \sum_{b=1}^{N} \boldsymbol{u}_{a} \cdot \mathrm{T}_{a b} \cdot \boldsymbol{u}_{b},
$$


where $\boldsymbol{u}_{a}$ is the deviation of the ath electron from its equilibrium position, $\boldsymbol{p}_{a}$ is its momentum and the dipolar tensor $T_{a b}$ is defined by

$$
\begin{aligned}
\mathrm{T}_{a b} & =\mathbf{V}_{a} \nabla_{b} \frac{1}{R_{a b}}=\frac{1}{R_{a b}^{3}}\left(1-3 \frac{\boldsymbol{R}_{a b} \boldsymbol{R}_{a b}}{R_{a b}^{2}}\right), \quad \text { if } a \neq b, \\
\mathrm{~T}_{a a} & =0,
\end{aligned}
$$

where $\boldsymbol{R}_{a b}=\boldsymbol{R}_{a}-\boldsymbol{R}_{b}$ and $\boldsymbol{R}_{a}$ and $\boldsymbol{R}_{b}$ are the equilibrium positions of the oscillators $a$ and $b$, respectively.

The equations of motion of the oscillators are

$$
m \ddot{u}_{a}+m \omega_{0}^{2} u_{a}=-e^{2} \sum_{b=1}^{N} \mathrm{~T}_{a b} \cdot u_{b} .
$$

Let $\lambda_{i}(i=1, \ldots, 3 N)$ be the eigenvalues of the $3 N \times 3 N$ matrix $\mathrm{T}$ built up from the $3 \times 3$ matrices $T_{a b}$. Then the eigenfrequencies of the interacting oscillator system are

$$
\Omega_{i}=\omega_{0}\left(1+\alpha \hat{\lambda}_{i}\right)^{\frac{1}{2}}
$$

where $\alpha=e^{2} / m \omega_{0}^{2}$ is the static polarizability of the atom.

For a particular configuration of the atoms the interaction energy $\Delta E_{0}$ in the electronic ground state of the system is found as the difference in zcro-point energy for this configuration and for the configuration where all the atoms are infinitely far apart:

$$
\begin{aligned}
1 E_{0} & =\frac{1}{2} \hbar \sum_{i}\left(\Omega_{i}-\omega_{0}\right)=\frac{1}{2} \hbar \omega_{0} \sum_{i}\left[\left(1+\alpha \hat{\lambda}_{i}\right)^{\frac{1}{2}}-1\right] \\
& =-\frac{1}{2} \hbar \omega_{0} \sum_{i} \sum_{n=1}^{\infty} c_{n}(-\alpha)^{n} \hat{\lambda}_{i}^{n}=-\frac{1}{2} \hbar \omega_{0} \sum_{n=2}^{\infty} c_{n}(-\alpha)^{n} \operatorname{Tr} \mathrm{T}^{n} \\
& =-\frac{1}{2} \hbar \omega_{0} \sum_{n=2}^{\infty} c_{n}(-\alpha)^{n} \sum_{a_{1}, \ldots, a_{n}=1}^{N} \operatorname{Tr}\left(\mathrm{T}_{a_{1} a_{2}} \cdot \mathrm{T}_{a_{2} a_{3}} \cdots \cdot \mathrm{T}_{a_{n} a_{1}}\right),
\end{aligned}
$$

where the expansion coefficient

$$
c_{n}=\frac{(2 n) !}{(2 n-1) n !^{2} 2^{2 n}}
$$

and $\operatorname{Tr}$ denotes the trace. Note that $\operatorname{Tr} \operatorname{T}=0$.

For $n=2$ :

$$
\operatorname{Tr} \mathrm{T}^{2}=\sum_{a \cdot b} \operatorname{Tr}\left(\mathrm{T}_{a b} \cdot \mathrm{T}_{b a}\right)=\sum_{a \neq b} 6 / R_{a b}^{6} .
$$


Hence the first term $n=2$ of the expansion (5) for $\Delta E_{0}$ leads to the approximate expression for the interaction energy:

$$
\Delta E_{0}^{(2)} \cong-\frac{3}{4} h \omega_{0} \alpha^{2} \sum_{a<b} 1 / R_{u h l}^{6},
$$

which is simply the sum of the Van der Waals pair interactions. The terms $n=3$, $n=4$, etc., give the contributions due to three-, four-, etc., particle interactions, or rather, to be more precise, the contributions due to three, four, etc., bonds, i.e. factors $\alpha \mathbf{T}_{a_{i} a_{k}}$. This is because the particles $a_{1}, a_{2}, \ldots, a_{n}$ in (5) need not be different; for instance the term $n=4$ contains in addition to four-particle also twoand three-particle interactions. The expression (5) has been derived independently by various authors ( $c f$. for instance refs. 1 and 2 ).

It follows from (5) that the binding energy per particle in an infinite system at zero temperature is given by

$$
U=\frac{1}{2} \hbar \omega_{0} \sum_{n=2}^{\infty} c_{n}(-\alpha)^{n} \sum_{a_{2}, \ldots, a_{n}=1}^{\infty}\left\langle\operatorname{Tr}\left(\mathrm{T}_{a_{1} a_{2}} \cdot \mathrm{T}_{a_{2} a_{3}} \cdots \mathrm{T}_{a_{2} a_{1}}\right)\right\rangle,
$$

where the brackets indicate an average over configurations. For ideal Bravais lattices (8) may be evaluated in the following way ${ }^{1}$ ): Introducing the sum over lattice points

$$
\mathrm{T}(\boldsymbol{k})=\sum_{\boldsymbol{R}_{n} \neq 0} \mathrm{~T}\left(\boldsymbol{R}_{n}\right) \mathrm{e}^{\mathrm{i} \boldsymbol{k} \cdot \boldsymbol{R}_{n}},
$$

which has three eigenvalues $\lambda_{\beta}(k),(\beta=1,2,3)$, we observe that

$$
\sum_{a_{2}, \ldots, a_{n}=1}^{\infty}\left\langle\operatorname{Tr}\left(\mathrm{T}_{a_{1} a_{2}} \cdot \mathrm{T}_{a_{2} a_{3}} \cdots \cdot \mathrm{T}_{a_{n} a_{1}}\right)\right\rangle=\left(1 / 8 \pi^{3} \varrho\right) \int_{B} \mathrm{~d}^{3} k \operatorname{Tr}[\operatorname{T}(k)]^{n},
$$

where $\varrho$ is the number density and the integration extends over the first Brillouin zone. Hence for this particular case $(8)$ reduces to

$$
\begin{aligned}
U & =\left(\hbar \omega o_{0} / 16 \pi^{3} \varrho\right) \sum_{n=2}^{\infty} c_{n}(-\alpha)^{n} \int_{B} \mathrm{~d}^{3} k \operatorname{Tr}[\mathrm{T}(k)]^{n} \\
& =-\left(h \omega_{0} / 16 \pi^{3} \varrho\right) \sum_{\beta} \int_{B} \mathrm{~d}^{3} k\left\{\left[1+\alpha \lambda_{\beta}(\boldsymbol{k})\right]^{\frac{1}{2}}-1\right\} .
\end{aligned}
$$

This expression has been evaluated numerically by Lucas ${ }^{1}$ ) for various noble-gas crystals by computing the eigenvalues $\lambda_{\beta}(\boldsymbol{k})$ in a large number of points in the first Brillouin zone. Here we reproduce his results for solid argon and xenon, which crystallize in the f.c.c. structure. Writing

$$
U=\frac{1}{2} h \omega_{0}, X=\frac{1}{2} h \omega_{0} \sum_{n=2}^{\infty} X_{n}
$$


where $X_{n}$ is the $n$-bond contribution, which is easily seen to be proportional to $z^{n}$ with

$$
z=4 \pi . \alpha,
$$

the values given in table I were obtained:

TABLE I

\begin{tabular}{|c|c|c|c|c|c|c|}
\hline & $z$ & $X \times 10^{3}$ & $X_{2} \times 10^{3}$ & $X_{3} \times 10^{3}$ & $X_{4} \times 10^{3}$ & $X_{5} \times 10^{3}$ \\
\hline Ar & 0.5196 & 8.8440 & 9.2572 & -0.5375 & 0.1420 & -0.0221 \\
\hline $\mathrm{Xe}$ & 0.8372 & 22.573 & 24.035 & -2.2479 & 0.9588 & -0.2390 \\
\hline
\end{tabular}

Note that the total contribution of 3-, 4-, etc., bond interactions (which alternate in sign) is of the order of $6 \%$ of the total binding energy.

Let us finish this introduction by making three remarks:

1) Expressions (8) for the binding energy per particle may be generalized to the case that an atom is represented by several oscillators with frequencies $\omega_{1}, \omega_{2}$, etc. One then finds ${ }^{3}$ )

$$
\begin{aligned}
U= & (h / 2 \pi) \sum_{n=2}^{\infty}\left[(-1)^{n} / n\right] \int_{0}^{\infty} \mathrm{d} \xi[x(\mathrm{i} \xi)]^{n} \\
& \times \sum_{a_{2}, \ldots, a_{n}=1}^{\infty}\left\langle\operatorname{Tr}\left(\mathrm{T}_{a_{1} a_{2}} \cdot \mathrm{T}_{a_{2} a_{3}} \cdots \cdot \mathrm{T}_{a_{n} a_{1}}\right)\right\rangle,
\end{aligned}
$$

where now $\alpha(\omega)=\left(e^{2} / m\right) \sum_{j} 1 /\left(\omega_{j}^{2}-\omega^{2}\right)$ is the dynamical polarizability of an atom.

2) Expression (8), respectively (12), have been used as a starting point to calculate the Van der Waals interaction between an atom at a distance $d$ from a dielectric halfspace and between two dielectric halfspaces at a distance $d$ apart, respectively ${ }^{3,4}$ ). On the assumption that $d$ is large compared to interatomic distances in the dielectric the sum of 2-, 3-, etc., bond interactions could be evaluated exactly and expressed in terms of the dielectric constant at imaginary frequencies of the dielectric medium, thus recovering the results obtained by Lifshitz ${ }^{5}$ ) previously in a macroscopic treatment of these problems. However, in the present case of one medium such a macroscopic treatment does not seem possible in a rigorous way.

3) As is well known, the Van der Waals interaction between two atoms or two oscillators is found correctly from the electrostatic dipole interaction as described by (1) only in the limit of small distance $\left(R \ll c /\left(\omega_{0}=\lambda_{0} / 2 \pi\right)\right.$ between the particles. For larger distances retardation effects have to be taken into account, 
which in the case $R \geqslant c / \omega_{0}$ lead to an $R^{-7}$ instead of an $R^{-6}$ dependence of the Van der Waals interaction energy ${ }^{6}$ ).

Also in the case of two macroscopic dielectrics at a distance $d$ apart, briefly discussed above, one must, when $d$ is no longer small compared to the wavelengths present in the atomic spectrum, consider the interaction with the full electromagnetic ficld in order to evaluate their Van der Waals interaction ${ }^{4,7}$ ). However. if one is interested in the binding energy of a dense dielectric medium, as we are at present, retardation corrections are completely negligible. This has been shown explicitly by Lucas $^{1}$ ) and may be understood by observing that the main contribution to the Van der Waals binding energy comes from atoms which are relatively close to each other. In this regard I also want to refer to the comprehensive but complicated work of Bullough ${ }^{8}$ ).

2. The long-nave approximation. We have seen in the introduction that for a crystal lattice the many-body Van der Waals binding energy can be evaluated exactly. For a fluid. however, this is no longer possible. It is true that, as follows from (7), the two-bond contribution to the binding energy per particle may be expressed in terms of the radial distribution function $g(r)$ :

$$
U^{(2)}=\frac{3}{3} h\left(r_{0} x^{2} 0 \int g(r)\left(1: r^{6}\right) \mathrm{d}^{3} r\right.
$$

but for the evaluation of 3-, 4-, etc., particle contributions one would need the 3-. 4-, ctc., particle distribution functions $[c f$. (8)] of which very little is known. Hence in the case of a fluid, approximations are unavoidable.

In this section we will discuss a particularly simple approximation, the so-called long-wave approximation (LWA), originally introduced by Doniach ${ }^{9}$ ) and also investigated by Lucas $^{1}$ ). In an ideal Bravais lattice the eigenmodes of the interacting oscillator system are plane waves (polarisation waves):

$$
\boldsymbol{u}_{n}=\boldsymbol{A} \mathrm{e}^{\mathrm{i}\left[k \cdot \boldsymbol{R}_{n}-(\omega)(k) t\right]} \text {, }
$$

for ach $k$ vector with three mutually orthogonal polarisations. The frequencies ${ }^{\prime \prime}{ }_{\beta}(k)(\beta=1,2,3)$ are given by

$$
{ }^{(1)}(k)=\omega_{0}\left[1+\curvearrowright \hat{\lambda}_{\beta}(k)\right]^{\frac{1}{2}},
$$

as may, e.g., be seen from (10). $\lambda_{\beta}(k)$ are the eigenvalues of the tensor $\mathrm{T}(\boldsymbol{k})$ introduced in (9). The LWA is now obtained by replacing in (10) for all values of $k$ in the Brillouin zone $\lambda_{\beta}(k)$ by $\lambda_{\beta}(0)$. In this long-wavelength limit the three polarisation waves reduce to one longitudinal and two degenerate transverse waves, the frequencies of which are given by (see, e.g., Lucas $\left.{ }^{1}\right)$ ):

$$
\omega_{1}=\omega_{0}(1+2 z / 3)^{\frac{1}{2}}, \quad \omega_{1}=\omega_{0}(1-z / 3)^{\frac{1}{2}} .
$$


where $z=4 \pi \rho \alpha[c f .(11)]$. Hence in the LWA the binding energy per particle is given by

$$
\begin{aligned}
U_{\mathrm{LWA}} & =-{ }_{2}^{1} h \omega_{0}\left[2(1-z / 3)^{\frac{1}{2}}+(1+2 z / 3)^{\frac{1}{2}}-3\right] \\
& =\left(h \omega_{0} z^{2} / 24\right)\left(1-\frac{1}{6} z+\frac{5}{48} z^{2}+\cdots\right) .
\end{aligned}
$$

The successive terms in this expansion give the contributions of 2-, 3-, 4-, etc., bond interactions.

I would like to stress that the LWA expression (16) may also be obtained from simple macroscopic considerations and as a consequence would be applicable to fluids as well as to crystals. The cquations of electrostatics (retardation neglected) for any dielectric

$$
\nabla \times E=0, \quad \nabla \cdot \varepsilon E=0,
$$

are easily seen to have the following solutions: 1) longitudinal polarisation waves with arbitrary wavevector $\boldsymbol{k}$ and with a frequency $\omega_{1}$ (independent of $\boldsymbol{k}$ ), such that $\varepsilon\left(\omega_{1}\right)=0$; and 2) two degenerate transverse polarisation waves with arbitrary wavevector $\boldsymbol{k}$ and with a frequency $\omega_{\mathrm{t}}$ (independent of $\boldsymbol{k}$ ), such that $\varepsilon\left(\omega_{\mathrm{t}}\right)=\infty$.

If for our model dielectric we now take the dependence of $\varepsilon$ on $\omega$ according to the Clausius-Mossotti equation

$$
\frac{\varepsilon(\omega)-1}{\varepsilon(\omega)+2}=\frac{4 \pi}{3} \varrho \alpha(\omega)=\frac{4 \pi}{3} g \frac{e^{2}}{m\left(\omega_{0}^{2}-\omega^{2}\right)},
$$

we are immediately led to expressions (15) for the longitudinal and transverse frequencies, respectively, bearing in mind that $\alpha \equiv \alpha(0)]$. For a system of $N$ atoms one should restrict the number of longitudinal and transverse polarisation modes to $N$ and $2 N$, respectively. The difference in zero-point energy between the actual system and a very dilute one then leads to the LWA binding energy (16).

TABLE II

\begin{tabular}{cccccc}
\hline & $z$ & $X_{\mathrm{LWA}} \times 10^{3}$ & $X_{2 \mathrm{LWA}} \times 10^{3}$ & $X_{3 \mathrm{LWA}} \times 10^{3}$ & $X_{4 \mathrm{LWA}} \times 10^{3}$ \\
\hline $\mathrm{Ar}$ & 0.5196 & 21.08 & 22.50 & -1.95 & 0.63 \\
$\mathrm{Xe}$ & 0.8372 & 53.60 & 58.41 & -8.15 & 4.26 \\
\hline
\end{tabular}

In order to judge the quality of the LWA we may evaluate the expression (16) for the case of the noble-gas crystals for which exact results (reproduced in table I) are available. The corresponding quantities in LWA are assembled in table II.

A comparison of tables I and II makes it clear that the LWA is rather bad for the noble-gas crystals. In particular the 2-bond contribution is much too large: 
$X_{2 \text { L.WA }} / X_{2} \approx 2.4$. The ratio of the many-bond contributions ( 3 and more) to the 2-bond contribution, however, is somewhat better; e.g., for solid argon this ratio is -0.063 in the LWA, while its exact value is -0.045 (for solid xenon the corresponding values are -0.082 and -0.061 , respectively).

3. The uniform-integration approximation. In the approximation to be discussed now we start from the basic (and exact) expression (8) for the binding energy per particle. However, instead of averaging over particle configurations with the proper distribution functions, we simply integrate uniformly over the positions of particles $3,4, \ldots, n$. Not, as will be explained below, over the position of particle 2 because this would lead to divergences [cf. e.g. (7)].

Thus in this uniform-integration approximation (UIA) we write:

$$
U_{\mathrm{UIA}}=\frac{1}{2} \hbar \omega_{0} \sum_{n=2}^{\infty} c_{n}(-\alpha)^{n} \varrho^{n-1} \int \operatorname{Tr}\left(\mathrm{T}_{12} \cdot \mathrm{T}_{23} \cdots \cdot \mathrm{T}_{n 1}\right) \mathrm{d}^{3} 2 \mathrm{~d}^{3} 3 \cdots \mathrm{d}^{3} n .
$$

The convolution integral appearing here may conveniently be transformed into an integral over Fourier space; i.e., if we introduce

$$
\hat{\mathrm{T}}(\boldsymbol{k})=\int \mathrm{T}(\boldsymbol{r}) \mathrm{e}^{\mathrm{i} k \cdot r} \mathrm{~d}^{3} r,
$$

we have

$$
U=\frac{1}{2} \hbar \omega_{0} \sum_{n=2}^{\infty} c_{n}(-\alpha \varrho)^{n}\left(1 / 8 \pi^{3} \varrho\right) \int \operatorname{Tr}[\hat{\mathrm{T}}(k)]^{n} \mathrm{~d}^{3} k .
$$

Now it may be shown easily, e.g., by partial integration, that

$$
\hat{\mathrm{T}}(\boldsymbol{k})=\frac{4}{3} \pi\left[\left(3 \boldsymbol{k} k / k^{2}\right)-1\right] .
$$

[Actually, in order to make $T(\boldsymbol{r})$ traceless everywhere one should define $T(\boldsymbol{r})$ $\left.\equiv-\nabla \nabla(1 / r)-\frac{4}{3} \pi 1 \delta(r).\right]$

The eigenvalues of the tensor $\hat{T}(\boldsymbol{k})$ are evidently $\frac{8}{3} \pi,-\frac{4}{3} \pi,-\frac{4}{3} \pi$ and therefore

$$
\operatorname{Tr}[\hat{\mathrm{T}}(\boldsymbol{k})]^{n}=\left(\frac{4}{3} \pi\right)^{n}\left[2^{n}+2(-1)^{n}\right]=\frac{1}{6}\left(\frac{4}{3} \pi\right)^{n-2}\left[2^{n}+2(-1)^{n}\right] \operatorname{Tr}[\hat{\mathrm{T}}(\boldsymbol{k})]^{2} .
$$

In (23) we have expressed $\operatorname{Tr}[\hat{\mathrm{T}}(\boldsymbol{k})]^{n}$ as a multiple of $\operatorname{Tr}[\hat{\mathrm{T}}(\boldsymbol{k})]^{2}$, that is, in the UIA we express the many-bond contributions in terms of the 2-bond contribution, which may be considered to be known.

Indeed, we should replace

$$
\left(1 / 8 \overline{-}^{3}\right) \int \operatorname{Tr}[\hat{\mathrm{T}}(k)]^{2} \mathrm{~d}^{3} k=\int \operatorname{Tr}\left(\mathrm{T}_{12} \cdot \mathrm{T}_{21}\right) \mathrm{d}^{3} 2,
$$

by

$$
6 \int g(r) r^{-6} \mathrm{~d}^{3} r=24 \pi \int_{0}^{\infty} g(r) r^{-4} \mathrm{~d} r
$$

where $g(r)$ is the radial distribution function of the fluid. 
Thus we find in this approximation from (21), (23) and (24)

$$
\begin{aligned}
U_{\mathrm{UIA}}= & \frac{1}{2} \hbar \omega_{0} \varrho^{-1}\left(\frac{4}{3} \pi\right)^{-2} \int g(r)\left(1 / r^{6}\right) \mathrm{d}^{3} r \\
& \times \sum_{n=2}^{\infty} c_{n}\left(-\frac{4}{3} \pi \alpha \varrho\right)^{n}\left[2^{n}+2(-1)^{n}\right] \\
= & \frac{1}{2} \hbar \omega_{0}\left(\frac{4}{3} \pi g\right)^{-2} \varrho \int g(r) r^{-6} \mathrm{~d}^{3} r\left[3-\left(1+\frac{2}{3} z\right)^{\frac{1}{2}}-2\left(1-\frac{1}{3} z\right)^{\frac{1}{2}}\right] \\
= & \frac{3}{8} \hbar \omega_{0} x^{2} \varrho \int g(r) r^{-6} \mathrm{~d}^{3} r\left(1-\frac{1}{6} z+\frac{5}{48} z^{2}+\cdots\right) .
\end{aligned}
$$

Comparison with (16) shows that, whereas in the present uniform-integration approximation the 2-bond contribution is treated exactly (in terms of the pair distribution function), the ratios of the 3-, 4-, etc., bond contributions to the 2-bond one are precisely the same as in the long-wave approximation. That means that the main defect of the LWA has been overcome. As a matter of fact (25) might for a check be applied to the noble-gas crystals as we did in the case of the LWA. Of course $g(\boldsymbol{r})$ here depends on direction and the integral in (25) reduces to a lattice sum. The 2-bond contribution is obviously given exactly, while from our discussion in section 2 it follows that the many-bond corrections, which anyhow are small, are too big in this case by a factor of the order of 1.4.

In this section we have substituted approximate expressions for the average traces in the basic formula (8). It will be obvious that we might make the same substitution in the more general formula (12). We will not write down the corresponding expressions however.

Let us add one further remark here. Recently the question of the correction factor due to many-particle interactions to the Van der Waals interaction between two atoms of a dense rare-gas fluid has received some attention ${ }^{10,11}$ ). In the clectrostatic oscillator model also used in the present paper the following expression was derived for the effective interaction between two atoms at separation $R_{i j}$ :

$$
\varphi_{i j}^{\text {eff }}=-\frac{3}{4}\left(\hbar \omega_{0} x^{2} / R_{i j}^{6}\right)\left(1-\frac{1}{6} z+\text { higher-order terms in } z\right) .
$$

In ref. 11 it was shown that the first-order correction $-\frac{1}{6} z$ is rigorously correct for large separation $R_{i j}\left[R_{i j}>2 R_{0}\right.$ where $R_{0}$ is a correlation distance such that $g(R)=1$ for $R>R_{0}$ ]. It may be clear that if one were to apply (26) for all interatomic distances one would obtain the result (25) for the binding energy $U$ with the same coefficient of the correction term in $z$.

4. Some alternative approximations. Let us consider again the expression $(n \geqslant 3)$

$$
\sum_{2,3, \ldots, n}\left\langle\operatorname{Tr}\left(\mathrm{T}_{12} \cdot \mathrm{T}_{23} \cdots \cdots \mathrm{T}_{n 1}\right)\right\rangle,
$$


to be cvaluated according to our starting point (8). Instead of integrating uniformly over the positions of the particles, as we did in section 3, we now approximate (27) by

$$
\varrho^{n-1} \int \operatorname{Tr}\left(g_{12} \mathrm{~T}_{12} \cdot g_{23} \mathrm{~T}_{23} \cdots \cdot g_{n 1} \mathrm{~T}_{n 1}\right) \mathrm{d}^{3} 2 \mathrm{~d}^{3} 3 \cdots \mathrm{d}^{3} n,
$$

where $g_{i j}=g\left(\boldsymbol{R}_{j}-\boldsymbol{R}_{i}\right)$ again is the pair distribution function. That is, we apply to the many-particle distribution functions a kind of generalized superposition approximation. (For $n=2$ this would not be reasonable because the function $g_{12}$ would be squared here.) If we now introduce the fourier transform

$$
G(k) \equiv \int g(r) \mathrm{T}(\boldsymbol{r}) \mathrm{e}^{\mathrm{i} k \cdot \boldsymbol{R}} \mathrm{d}^{3} r .
$$

we can write (28) as

$$
\left(0^{n-1} / 8 \pi^{3}\right) \int \operatorname{Tr}[\mathrm{G}(k)]^{n} \mathrm{~d}^{3} k .
$$

Notice that (30) would be correct for a crystal, except that in this case the integration over $k$ space should be restricted to the first Brillouin zone as a consequence of the periodicity $[c f .(10)]$. For a fluid $g(\boldsymbol{r})=g(r)$ and then it is not difficult to show that ${ }^{12}$ )

$$
\mathrm{G}(\boldsymbol{k}) \equiv \int g(r) \mathrm{T}(\boldsymbol{r}) \mathrm{e}^{\mathrm{i} k \cdot \boldsymbol{R}} \mathrm{d}^{3} r=\mu(k)\left[\left(3 k \boldsymbol{k} / k^{2}\right)-1\right],
$$

with

$$
\mu(k)=(2 \pi)^{3 / 2} k^{-\frac{1}{2}} \int_{0}^{\infty} g(r) J_{5 / 2}(k r) r^{-3 / 2} \mathrm{~d} r,
$$

where $J_{5 / 2}$ is a Bessel function. Notice that for $g(r) \equiv 1, \mu(k)=\frac{4}{3} \pi[c f .(22)]$, and also that $\lim _{k \rightarrow 0} \mu(k)=\frac{4}{3} \pi$.

In the same way as in section 3 we now find that

$$
\operatorname{Tr}[G(k)]^{n}=\left[2^{n}+2(-1)^{n}\right] \mu^{n}(k)
$$

and from that

$$
\begin{aligned}
U= & \frac{1}{16} \hbar\left(\omega_{0} \alpha^{2} \sum_{2}\left\langle\operatorname{Tr} \mathrm{T}_{12} \cdot \mathrm{T}_{21}\right\rangle\right. \\
& +\frac{\hbar \omega_{0}}{16 \pi^{3} \varrho} \sum_{n=3}^{\infty} c_{n}(-\alpha \varrho)^{n}\left[2^{n}+2(-1)^{n}\right] \int \mu^{n}(k) \mathrm{d}^{3} k \\
= & \frac{3}{8} \hbar \omega_{0} \alpha^{2} \sum_{2}\left\langle\frac{1}{R_{12}^{6}}\right\rangle+\frac{\hbar \omega_{0}}{16 \pi^{3} \varrho} \\
& \times \int\left\{3-2[1-\alpha \varrho \mu(k)]^{\frac{1}{2}}-[1+2 \times \varrho \mu(k)]^{\frac{1}{2}}-\frac{3}{4} x^{2} \varrho^{2} \mu^{2}(k)\right\} \mathrm{d}^{3} k
\end{aligned}
$$




$$
\begin{aligned}
= & \frac{3}{8} h \omega \omega_{0} x^{2} \varrho \int g(r) r^{-6} \mathrm{~d}^{3} r \\
& +\frac{\hbar \omega_{0}}{16 \pi^{3} \varrho} \int\left\{-\frac{3}{4}[\alpha \varrho \mu(k)]^{3}+\frac{45}{64}[\alpha \varrho \mu(k)]^{4}-\cdots\right\} \mathrm{d}^{3} k .
\end{aligned}
$$

In this approximation, as in (25), the structure of the fluid is taken into account through the intermediary of its radial distribution function only. Here, however, the many-bond interactions give rise to an additive term in the binding energy, whereas in (25) they yield a correction factor.

A rather similar result may be obtained in a different way. Remember the equations of motion of the oscillators given by (3). Now for a fluid let us write the equation of motion of the oscillator at the origin of coordinate system as:

$$
m \ddot{\boldsymbol{u}}+m \omega_{0}^{2} \boldsymbol{u}=-e^{2} \varrho \int g(r) \mathrm{T}(r) \cdot \boldsymbol{u}(\boldsymbol{r}) \mathrm{d}^{3} r,
$$

i.e., by way of approximation we replace the actual surroundings of an atom by its average surroundings. Thus the equations of motion have been made translationally invariant and, as in a crystal lattice, have as solutions plane polarisation waves

$$
\boldsymbol{u}(r)=A \mathrm{e}^{\mathrm{i}[k \cdot R-\omega(k) \imath]} .
$$

Substituting this expression in (35) one verifies with the help of (29) and (31), that for every wave-vector $\boldsymbol{k}$ one obtains a longitudinal wave with frequency

$$
\omega_{!}(k)=\omega_{0}[1+2 \alpha \varrho \mu(k)]^{\frac{1}{2}}
$$

and two degenerate transverse waves with frequency

$$
\omega_{t}(k)=\omega_{0}[1-\alpha \varrho \mu(k)]^{\frac{1}{2}},
$$

where $\mu(k)$ is given by (32).

As in Debye's theory of specific heat, because of the atomic structure of the dielectric medium we restrict the domain of $\boldsymbol{k}$ values to a sphere $S$ of radius $k_{\mathrm{m}}$ such that

$$
\left(1 / 8 \pi^{3} \varrho\right) \int_{S} \mathrm{~d}^{3} k=1, \quad \text { i.e., } \quad k_{\mathrm{m}}=\left(6 \pi^{2} \varrho\right)^{1 / 3} .
$$

For the binding energy per particle we then find:

$$
U=\frac{\hbar \omega_{0}}{16 \pi^{3} \varrho} \int_{S}\left\{3-2[1-\alpha \varrho \mu(k)]^{\frac{1}{2}}-[1+2 \alpha \varrho \mu(k)]^{\frac{1}{1}}\right\} \mathrm{d}^{3} k .
$$


If in (39) we were to replace $\mu(k)$ by $\mu(0)=\frac{4}{3} \pi$ for all values of $k$ inside the sphere $S$, we should recover the result (16) of the long-wave approximation. Of course in (39) the 2-, 3-, 4-, elc., bond contributions are all treated approximately. But we have an exact expression for the 2-bond contribution. Therefore it seems advisable to leave out in (39) the quadratic term in $\alpha$ o and replace it by the exact 2-bond term. We then find:

$$
\begin{aligned}
U= & \frac{3}{8} \hbar \omega_{0} \alpha^{2} \varrho \int g(r) r^{-6} \mathrm{~d}^{3} r \\
& +\frac{h \omega_{0}}{16 \pi^{3} \varrho} \int_{s}\left\{-\frac{3}{8}[x \varrho \mu(k)]^{3}+\frac{45}{64}[x \varrho \mu(k)]^{4}-\cdots\right\} \mathrm{d}^{3} k \\
= & \frac{3}{8} h \omega_{0} \alpha^{2} \varrho \int_{\left(6 \pi^{2} \varrho\right)^{1 / 3}} g(r) r^{-6} \mathrm{~d}^{3} r \\
& +\frac{\hbar \omega_{0}}{4 \pi^{2} \varrho} \int_{0}^{k^{2} \mathrm{~d} k\left\{-\frac{3}{8}[\alpha \varrho \mu(k)]^{3}+\frac{45}{64}[\alpha \varrho \mu(k)]^{4}-\cdots\right\}} .
\end{aligned}
$$

Note that this way we have reached the same result as in (34), except that now the integration over $k$ values is restricted to the sphere $S$.

Alternatively, we might in (39) write the 2-bond contribution [the quadratic term in $\alpha e \mu(k)]$ as a factor in front of the expression and then replace it by the exact 2-bond contribution. That way we oblain

$$
\begin{aligned}
U= & \left(\frac{1}{2} h \omega \varrho_{0} \varrho^{-1} \int g(r) r^{-6} \mathrm{~d}^{3} r / \int_{s}[\mu(k)]^{2} \mathrm{~d}^{3} k\right) \\
& \times \int_{S}\left\{3-2[1-\alpha \varrho \mu(k)]^{\frac{1}{2}}-[1+2 \lambda \varrho \mu(k)]^{\frac{1}{2}}\right\} \mathrm{d}^{3} k .
\end{aligned}
$$

If here we were to replace $\mu(k)$ by $\mu(0)={ }_{3}^{4} \pi$ we should recover the result $(25)$ of the uniform-integration approximation.

5. Discussion. In the present paper we have discussed the many-body Van der Waals binding energy in a dense system of neutral atoms. We have represented an atom by one (or more) harmonic oscillators (Drude-Lorentz model) and we have assumed that the atoms interact through electrostatic dipole-dipole forces only. Retardation cffects may be cxpected to be negligible, because the binding energy is determined by clusters of atoms which are relatively close.

In the case of crystal lattices the many-body contribution to the binding energy can be evaluated exactly ( $c f$. section 1). For the solid noble gases the 3-, and morebond contributions are small, taken together they are of the order of $6 \%$ of the 
total binding energy. For a fluid the main 2-bond contribution may be expressed exactly in terms of the radial distribution function $g(r)$. For an exact evaluation of the many-bond contributions (which again will give a small correction only) one would need the many-particle distribution functions. We have derived a number of approximate expressions for the many-bond corrections to the binding energy. They are given by (25), (34), (40) and (41), respectively. In (34) and (40) the 3-, 4-, etc., bond contributions result in an additive correction to the binding energy, in (25) and (41) they give rise to a correction factor to the 2-bond contribution.

It is difficult to assess the accuracy of these approximate expressions, because for a fluid an exact result is not available. As was discussed in section 3, the simple result (25), when applied to the solid noble gases, overestimates the many-bond correction by a factor of order 1.4. The methods employed in section 4 , which lead to (34), (40) and (41) respectively, on the other hand would in principle be correct for crystal lattices. Moreover, they do not make use of any long-wave approximation. For the moment I would think that the result (40) [or possibly (41)] would yield the most satisfactory approximation.

The 2-bond binding energy that would follow from (39) is

$$
\left(3 / 16 \pi^{2}\right) \hbar \omega_{0} x^{2} 0 \int_{0}^{\left(6 \pi^{2} \rho\right)^{1 / 3}} k^{2} \mu(k)^{2} \mathrm{~d} k,
$$

while the exact 2-bond binding energy is

$$
\frac{3}{2} \pi \hbar \omega_{0} x^{2} \sigma \int_{0}^{\infty} g(r) r^{-4} \mathrm{~d} r
$$

These expressions might be evaluated and compared for a realistic $g(r)$ function, for instance that of liquid argon. If the results would turn out to be relatively close, the approximations (39), (40) and (41) would obviously be equivalent. In this way it would also be possible to verify whether for $n \geqslant 3$

$$
\int_{S}[\mu(k)]^{n} \mathrm{~d}^{3} k
$$

would come very close to the corresponding unrestricted integration. If so the approximations (34) and (40) would be equivalent. We intend to investigate these points in the near future.

I would like to make one further remark. The present theory breaks down as soon as two (or more) atoms come too close together. This may be seen from the expression (4) for the eigenfrequencies of the coupled oscillator system. In order that these eigenfrequencies be real we must require that $\alpha \lambda_{i} \geqslant-1$. Now for a system of two particles at distance $r$ the 6 values of $\lambda_{i}$ are easily seen to be $\pm 2 / r^{3}$, $\pm 1 / r^{3}, \pm 1 / r^{3}$. The 2-particle (in contradistinction to the 2-bond) contribution to 
the binding energy per particle in a large system of particles is therefore

$$
\begin{aligned}
& -\frac{1}{2} \hbar \omega_{0}\left(\varrho \int g ( r ) \left[\frac{1}{2}\left(1+\frac{2 \alpha}{r^{3}}\right)^{\frac{1}{2}}+\frac{1}{2}\left(1-\frac{2 \alpha}{r^{3}}\right)^{\frac{1}{2}}+\left(1+\frac{\alpha}{r^{3}}\right)^{\frac{1}{2}}\right.\right. \\
& \left.\left.+\left(1-\frac{\alpha}{r^{3}}\right)^{\frac{3}{2}}-3\right] \mathrm{~d}^{3} r\right) .
\end{aligned}
$$

In this expression the contribution of an arbitrary (but even) number of bonds between two particles is included. For this contribution to the binding energy to be real we must require that distances between particles such that $r^{3}<2 \alpha$ do not occur, i.e., the particles must have a hard core with a diameter not smaller than $(2 \alpha)^{1 / 3}$. For a cluster of three particles (at equal distance $r$ ) one finds that $r^{3}$ should be larger than $5 / 2 \alpha$. The same value $5 / 2 \alpha$ is found for a cluster of four particles arranged in the vertices of a regular tetrahedron. For clusters of more particles the calculation of the eigenvalues $\lambda_{i}$ becomes rather difficult, but it seems safe to conclude that the theory is consistent as long as the distance of closest approach between atoms is larger than two times $x^{1 / 3}$, which for a substance like argon for instance is still rather smaller than the diameter of the atoms (for an argon atom $\alpha$ is about $1.65 \AA^{3}$ ).

A similar situation arises in the usual theories of the dielectric constant, which again break down in case two (or more) atoms are allowed to come too close. I hope to discuss this problem in another publication.

Acknowledgements. With pleasure I acknowledge useful discussions with my colleagues Professors N. G. van Kampen and Th. W. Ruijgrok.

\section{RLFLRENCLS}

1) Lucas, A., Physica 35 (1967) 353, thesis, Liege, 1966.

2) Renne, M.J. and Nijboer, B. R.A., Chem. Phys. Letters 1 (1967) 317.

3) Nijboer, B.R.A. and Renne, M.J., Chem. Phys. Letlers 2 (1968) 35.

4) Renne, M.J., thesis, Utrecht, 1971.

5) Litshitz, E. M., Soviet Physics-JE'TP 2 (1956) 73.

$C f$. also Landau, L. and Lifshilz, E. M., Electrodynamics of Continuous Media, Pergamon Press (Oxford, 1960) section 90.

6) Casimir, H.B.G. and Polder, D., Phys. Rev. 73 (1948) 360.

7) Renne, M. J., Physica 53 (1971) 193; 56 (1971) 125.

8) Bullough, R. K., J. Phys. A 2 (1969) 477.

9) Doniach, S., Phil. Mag. 8 (1963) 129.

10) Robinson, G. and March, N.H., J. Phys. C 5 (1972) 2553.

11) Renne, M.J. and Nijboer, B. R. A., J. Phys. C 6 (1973) L.10.

12) Cf. for instance: Sneddon, I.N., Fourier Transforms, McGraw-Hill (New York, 1951) p. $366 \mathrm{ff}$. 